PROCEEDINGS OF THE

AMERICAN MATHEMATICAL SOCIETY

Volume 140, Number 8, August 2012, Pages 2721-2730

S 0002-9939(2011)11272-3

Article electronically published on November 28, 2011

\title{
EMBEDDABILITY OF LOCALLY FINITE METRIC SPACES INTO BANACH SPACES IS FINITELY DETERMINED
}

\author{
M. I. OSTROVSKII
}

(Communicated by Thomas Schlumprecht)

This paper is dedicated to the memory of Nigel J. Kalton

Abstract. The main purpose of the paper is to prove the following results:

- Let $A$ be a locally finite metric space whose finite subsets admit uniformly bilipschitz embeddings into a Banach space $X$. Then $A$ admits a bilipschitz embedding into $X$.

- Let $A$ be a locally finite metric space whose finite subsets admit uniformly coarse embeddings into a Banach space $X$. Then $A$ admits a coarse embedding into $X$.

These results generalize previously known results of the same type due to Brown-Guentner (2005), Baudier (2007), Baudier-Lancien (2008), and the author $(2006,2009)$.

One of the main steps in the proof is: each locally finite subset of an ultraproduct $X^{\mathcal{U}}$ admits a bilipschitz embedding into $X$. We explain how this result can be used to prove analogues of the main results for other classes of embeddings.

\section{INTRODUCTION}

First we introduce necessary definitions:

Definition 1.1. A metric space $\left(A, d_{A}\right)$ is called discrete if there exists a constant $\delta>0$ such that $\forall u, v \in A \quad d_{A}(u, v) \geq \delta$. A discrete metric space $A$ is called locally finite if for every $u \in A$ and every $r>0$ the set $\left\{a \in A: d_{A}(u, a) \leq r\right\}$ is finite.

Let $C<\infty$. A map $f:\left(A, d_{A}\right) \rightarrow\left(Y, d_{Y}\right)$ between two metric spaces is called C-Lipschitz if

$$
\forall u, v \in A \quad d_{Y}(f(u), f(v)) \leq C d_{A}(u, v) .
$$

A map $f$ is called Lipschitz if it is $C$-Lipschitz for some $C<\infty$. For a Lipschitz map $f$ we define its Lipschitz constant by

$$
\operatorname{Lip} f:=\sup _{d_{A}(u, v) \neq 0} \frac{d_{Y}(f(u), f(v))}{d_{A}(u, v)} .
$$

A map $f: A \rightarrow Y$ is called a $C$-bilipschitz embedding if there exists $r>0$ such that

$$
\forall u, v \in A \quad r d_{A}(u, v) \leq d_{Y}(f(u), f(v)) \leq r C d_{A}(u, v) .
$$

Received by the editors March 3, 2011

2010 Mathematics Subject Classification. Primary 46B85; Secondary 05C12, 46B08, 46B20, $54 \mathrm{E} 35$.

Key words and phrases. Banach space, bilipschitz embedding, coarse embedding, locally finite metric space.

(c)2011 American Mathematical Society

Reverts to public domain 28 years from publication 
A bilipschitz embedding is an embedding which is $C$-bilipschitz for some $C<\infty$. The smallest constant $C$ for which there exist $r>0$ such that (10) is satisfied is called the distortion of $f$. A sequence of embeddings is called uniformly bilipschitz if they have uniformly bounded distortions.

A map $f:\left(X, d_{X}\right) \rightarrow\left(Y, d_{Y}\right)$ between two metric spaces is called a coarse embedding if there exist nondecreasing functions $\rho_{1}, \rho_{2}:[0, \infty) \rightarrow[0, \infty)$ (observe that this condition implies that $\rho_{2}$ has finite values) such that $\lim _{t \rightarrow \infty} \rho_{1}(t)=\infty$ and

$$
\forall u, v \in X \rho_{1}\left(d_{X}(u, v)\right) \leq d_{Y}(f(u), f(v)) \leq \rho_{2}\left(d_{X}(u, v)\right) .
$$

A sequence of embeddings is called uniformly coarse if all of them satisfy (2) with the same $\rho_{1}$ and $\rho_{2}$.

The main purpose of this paper is to prove the following two results:

Theorem 1.2. Let $A$ be a locally finite metric space whose finite subsets admit uniformly bilipschitz embeddings into a Banach space $X$. Then $A$ admits a bilipschitz embedding into $X$.

Theorem 1.3. Let $A$ be a locally finite metric space whose finite subsets admit uniformly coarse embeddings into a Banach space $X$. Then $A$ admits a coarse embedding into $X$.

Remark 1.4. It is worth mentioning that our argument implies that a similar result holds for any class $\mathcal{E}$ of embeddings provided that:

(a) There is a notion of being uniformly in $\mathcal{E}$ for a collection of maps of finite metric spaces into a Banach space.

(b) The notion in (a) is such that if all finite subspaces of a metric space $A$ admit uniformly-in- $\mathcal{E}$ embeddings into a Banach space $X$, then there is an embedding of the class $\mathcal{E}$ of $A$ into an ultraproduct $X^{\mathcal{U}}$, where $\mathcal{U}$ is a non-trivial ultrafilter (see the construction below).

(c) The image of a locally finite metric space under an embedding of the class $\mathcal{E}$ is locally finite.

(d) A composition of an embedding of the class $\mathcal{E}$ and a bilipschitz embedding is in $\mathcal{E}$.

Theorems 1.2 and 1.3 generalize previous results of the same type obtained in Bau07, BL08, BG05, Ost06a, Ost06b, Ost09; see section 4

Our proof uses: (a) the method of pasting embeddings of "pieces" suggested in BL08, (b) approaches to selection of basic subsequences developed in KP65, (c) some basic ultraproduct techniques going back to DK72.

\section{Proof in the BILIPSCHItZ CASE}

Proof of Theorem 1.2. We pick a point $O$ in $A$ and let $A_{i}=\left\{a \in A: d_{A}(O, a) \leq\right.$ $\left.2^{i}\right\}$. By the assumption there are uniformly bilipschitz maps $f_{i}: A_{i} \rightarrow X$. We may and shall assume that $f_{i}(O)=0$ and that there is a constant $1 \leq C<\infty$ such that

$$
\forall u, v \in A_{i} \quad d_{A}(u, v) \leq\left\|f_{i}(u)-f_{i}(v)\right\| \leq C d_{A}(u, v) .
$$

We are going to use some basic facts about ultraproducts of Banach spaces introduced in [DK72]. We refer to [DJT95, Chapter 8] for background on this matter. 
Let $\mathcal{U}$ be a nontrivial ultrafilter on $\mathbb{N}$. The maps $\left\{f_{i}\right\}_{i=1}^{\infty}$ induce a map $f: A \rightarrow$ $X^{\mathcal{U}}$ defined by $f(u)=\left\{\widetilde{f}_{i}(u)\right\}_{i=1}^{\infty}$, where

$$
\tilde{f}_{i}(u)= \begin{cases}f_{i}(u) & \text { if } u \in A_{i}, \\ 0 & \text { if } u \notin A_{i} .\end{cases}
$$

The definition of an ultraproduct immediately implies that $f: A \rightarrow X^{\mathcal{U}}$ is a bilipschitz embedding. Let $N=f(A)$. Since the composition of two bilipschitz embeddings is a bilipschitz embedding, it suffices to find a bilipschitz embedding of $N$ (with the metric induced from $X^{\mathcal{U}}$ ) into $X$.

Note. This passage from $A$ to its image in $X^{\mathcal{U}}$ is not essential for the proof of Theorem 1.2, it just simplifies some formulas in our proof. A similar step is more essential for other classes of embeddings.

Remark 2.1. We would like to emphasize that the rest of the proof of Theorem 1.2 consists of establishing the fact that a locally finite metric subspace of $X^{\mathcal{U}}$ admits a bilipschitz embedding into $X$.

Observation 2.2. If $X$ is finite-dimensional, then $X^{\mathcal{U}}$ is of the same dimension (see [DJT95, Proposition 8.4]), and the proof is completed.

If $X=L_{p}(0,1)$ for some $p \in[1, \infty]$, then each separable subspace of $X^{\mathcal{U}}$ is isometric to a subspace of $X$ (see [DJT95, Theorem 8.7] and the references in Ost09, p. 169]), so the proof is completed in this case, too.

In this connection in the rest of the proof we assume that $X$ is infinite-dimensional.

Let $N_{i}=\left\{u \in N:\|u\|_{X^{u}} \leq 2^{i}\right\}$. It is clear that the $N_{i}$ are finite sets. Using the same argument as in the proof of finite representability of $X^{\mathcal{U}}$ in $X$ (see DJT95, Theorem 8.13]) we get that there exist maps $s_{i}: N_{i} \rightarrow X$ such that $s_{i}(0)=0$ and

$$
\forall u, v \in N_{i} \quad\|u-v\| \leq\left\|s_{i}(u)-s_{i}(v)\right\| \leq\left(1+\frac{1}{i}\right)\|u-v\| .
$$

Since the sets $N_{i}$ form an increasing sequence, any subsequence $\left\{s_{n_{i}}\right\}_{i=1}^{\infty}$ of $\left\{s_{i}\right\}_{i=1}^{\infty}$ maps $\left\{N_{i}\right\}_{i=1}^{\infty}$ into $X$ and satisfies (3). We are going to construct a bilipschitz embedding of $N$ into $X$ using such subsequences.

Note. We are going to pass to a subsequence in $\left\{s_{i}\right\}_{i=1}^{\infty}$ several times. Each time we keep the notation $\left\{s_{i}\right\}_{i=1}^{\infty}$ for the subsequence.

Recall that a subspace $M \subset X^{*}$ is called 1-norming if

$$
\forall x \in X \sup \{|f(x)|: f \in M,\|f\| \leq 1\}=\|x\| .
$$

It is clear that we may assume that $X$ is separable (replacing it by the closure of the linear span of $\bigcup_{i=1}^{\infty} s_{i}\left(N_{i}\right)$, if necessary).

For a separable Banach space $X$ there exists a separable 1-norming subspace $M \subset X^{*}$. It can be constructed as follows: Let $\left\{x_{i}\right\}_{i=1}^{\infty}$ be a dense sequence in the unit sphere $S_{X}=\{x \in X:\|x\|=1\}$. Let $f_{i} \in S_{X^{*}}$ be such that $f_{i}\left(x_{i}\right)=1$. It is easy to check that the closed linear span $M$ of the sequence $\left\{f_{i}\right\}_{i=1}^{\infty}$ is 1-norming.

Let $M \subset X^{*}$ be a separable 1-norming subspace. Then the natural embedding of $X$ into $M^{*}$ is an isometry. We identify $X$ with its image under this embedding. Since $M$ is separable, there is a subsequence in $\left\{s_{i}\right\}$ such that the sequence 
$\left\{s_{i}(a)\right\}_{i=k}^{\infty}$ is convergent in the weak* topology of $M^{*}$ for each $a \in N_{k}$. We denote the weak* limit of this sequence by $m(a)$.

We need to select further subsequences of $\left\{s_{i}\right\}$. We do this in the following two steps.

Step 1. If for some $a, b \in N_{i}$ and some $j \geq i$ the vector $\left(s_{j}(a)-s_{j}(b)\right)-(m(a)-m(b))$ is nonzero, we find and fix $f=f_{j, a, b} \in S_{M}$ such that

$$
f\left(\left(s_{j}(a)-s_{j}(b)\right)-(m(a)-m(b))\right) \geq \frac{99}{100}\left\|\left(s_{j}(a)-s_{j}(b)\right)-(m(a)-m(b))\right\| .
$$

In such a case we assume that for all $k>j$ the condition

$$
\left|f\left(\left(s_{k}(a)-s_{k}(b)\right)-(m(a)-m(b))\right)\right| \leq \frac{1}{1000}|| a-b \|
$$

holds. This goal can be achieved because there are finitely many $a, b \in N_{i}$ and because $s_{k}(a)$ converges to $m(a)$ in the weak* topology of $M^{*}$.

Step 2. If $m(a) \neq m(b)$ for $a, b \in N_{i}$, we find and fix $f=f_{a, b} \in S_{M}$ such that

$$
f(m(a)-m(b)) \geq \frac{99}{100}\|m(a)-m(b)\|
$$

and select a subsequence satisfying

$$
\left|f\left(\left(s_{k}(a)-s_{k}(b)\right)-(m(a)-m(b))\right)\right| \leq \frac{1}{100}\|m(a)-m(b)\|
$$

for $k \geq i$. This can be achieved because $N_{i}$ is finite and $s_{k}(a)$ converges to $m(a)$ in the weak* topology of $M^{*}$.

We introduce a map $\varphi: N \rightarrow X$ by

$$
\varphi(a)=\frac{2^{i}-\|a\|}{2^{i-1}} s_{i}(a)+\frac{\|a\|-2^{i-1}}{2^{i-1}} s_{i+1}(a)
$$

if $2^{i-1} \leq\|a\| \leq 2^{i}$. One can easily check that the map is well-defined for $\|a\|=2^{i}$. Also we may assume that $\|a\| \geq 1$ for all $a \neq 0$, and so that each $a \neq 0$ satisfies $2^{i-1} \leq\|a\| \leq 2^{i}$ for some $i \in \mathbb{N}$.

We start by considering the case where $X$ is isomorphic to its hyperplane, and therefore $X$ is isomorphic to $X \oplus \mathbb{R}$. In this case we show that the embedding $\widetilde{\varphi}: N \rightarrow X \oplus \mathbb{R}$ given by $\widetilde{\varphi}(a)=(\varphi(a),\|a\|)$ is a bilipschitz embedding.

The proof in the case when $X$ is not isomorphic to its hyperplane is completed in section 2.4. (We know, by results of GM93, that spaces which are not isomorphic to their hyperplanes exist.)

Now we estimate the Lipschitz constants of $\widetilde{\varphi}$ and $(\widetilde{\varphi})^{-1}$. We consider three cases.

2.1. Case 1: $2^{i-1} \leq\|b\| \leq\|a\| \leq 2^{i}$. In this case we have

$$
\begin{aligned}
\varphi(a)-\varphi(b)= & \frac{2^{i}-\|a\|}{2^{i-1}} s_{i}(a)+\frac{\|a\|-2^{i-1}}{2^{i-1}} s_{i+1}(a) \\
& -\frac{2^{i}-\|b\|}{2^{i-1}} s_{i}(b)-\frac{\|b\|-2^{i-1}}{2^{i-1}} s_{i+1}(b) \\
= & \frac{2^{i}-\|a\|}{2^{i-1}}\left(s_{i}(a)-s_{i}(b)\right)+\frac{\|a\|-2^{i-1}}{2^{i-1}}\left(s_{i+1}(a)-s_{i+1}(b)\right) \\
& +\frac{\|b\|-\|a\|}{2^{i-1}} s_{i}(b)+\frac{\|a\|-\|b\|}{2^{i-1}} s_{i+1}(b) .
\end{aligned}
$$


Using (3) we get

$$
\begin{gathered}
\|\varphi(a)-\varphi(b)\| \leq \frac{2^{i}-\|a\|}{2^{i-1}} 2\|a-b\|+\frac{\|a\|-2^{i-1}}{2^{i-1}} 2\|a-b\| \\
+4|\|b\|-\|a\||+4|\|a\|-\|b\||
\end{gathered}
$$

and the fact that $\widetilde{\varphi}$ is a Lipschitz map is immediate.

Now we estimate the Lipschitz constant of $(\widetilde{\varphi})^{-1}$. So we need to estimate $\| \varphi(a)-$ $\varphi(b) \|$ from below. Observe that

$$
\begin{aligned}
\varphi(a)-\varphi(b)= & m(a)-m(b) \\
& +\frac{2^{i}-\|a\|}{2^{i-1}}\left(s_{i}(a)-s_{i}(b)-(m(a)-m(b))\right) \\
& +\frac{\|a\|-2^{i-1}}{2^{i-1}}\left(s_{i+1}(a)-s_{i+1}(b)-(m(a)-m(b))\right) \\
& +(\|b\|-\|a\|) \cdot \frac{s_{i}(b)}{2^{i-1}}+(\|a\|-\|b\|) \frac{s_{i+1}(b)}{2^{i-1}} .
\end{aligned}
$$

First we consider the case when $\|m(a)-m(b)\| \geq \frac{1}{100}\|a-b\|$. Let $f_{a, b}$ be the corresponding functional (see Step 2 above). We have

$$
\begin{aligned}
\|\varphi(a)-\varphi(b)\| \geq & f_{a, b}(\varphi(a)-\varphi(b)) \\
= & f_{a, b}(m(a)-m(b)) \\
& +f_{a, b}\left(\frac{2^{i}-\|a\|}{2^{i-1}}\left(s_{i}(a)-s_{i}(b)-(m(a)-m(b))\right)\right) \\
& +f_{a, b}\left(\frac{\|a\|-2^{i-1}}{2^{i-1}}\left(s_{i+1}(a)-s_{i+1}(b)-(m(a)-m(b))\right)\right) \\
& +(\|b\|-\|a\|) \cdot \frac{f_{a, b}\left(s_{i}(b)\right)}{2^{i-1}}+(\|a\|-\|b\|) \cdot \frac{f_{a, b}\left(s_{i+1}(b)\right)}{2^{i-1}} \\
& \geq \frac{99}{100}\|m(a)-m(b)\|-\frac{1}{100}\|m(a)-m(b)\| \\
& \quad-4|\|b\|-\|a\||-4|\|a\|-\|b\|| \\
\geq & \frac{98}{10000}\|a-b\|-8(\|a\|-\|b\|) .
\end{aligned}
$$

In the case when $\|a\|-\|b\|<\frac{1}{1000}\|a-b\|$, we get an estimate for the $\operatorname{Lip}\left(\varphi^{-1}\right)$ (and thus for $\left.\operatorname{Lip}\left((\widetilde{\varphi})^{-1}\right)\right)$ from above.

The estimate for $\operatorname{Lip}\left((\widetilde{\varphi})^{-1}\right)$ in the case when $\|a\|-\|b\| \geq \frac{1}{1000}\|a-b\|$ is immediate. We just recall that

$$
\widetilde{\varphi}(a)-\widetilde{\varphi}(b)=(\varphi(a)-\varphi(b)) \oplus(\|a\|-\|b\|) .
$$

Hence, to finish the estimate for $\operatorname{Lip}\left((\widetilde{\varphi})^{-1}\right)$ in Case 1 it remains to consider the case when $\|m(a)-m(b)\|<\frac{1}{100}\|a-b\|$. In this case we consider two subcases:

$$
\begin{aligned}
& \frac{2^{i}-\|a\|}{2^{i-1}}\left\|s_{i}(a)-s_{i}(b)-(m(a)-m(b))\right\| \geq \frac{1}{10}\|a-b\|, \\
& \frac{2^{i}-\|a\|}{2^{i-1}}\left\|s_{i}(a)-s_{i}(b)-(m(a)-m(b))\right\|<\frac{1}{10}\|a-b\| .
\end{aligned}
$$


We start with subcase (12). Let $f_{i, a, b}$ be the functional found in Step 1. We get

$$
\begin{aligned}
\|\varphi(a)-\varphi(b)\| \geq & f_{i, a, b}(\varphi(a)-\varphi(b)) \\
\geq & f_{i, a, b}(m(a)-m(b)) \\
& +f_{i, a, b}\left(\frac{2^{i}-\|a\|}{2^{i-1}}\left(s_{i}(a)-s_{i}(b)-(m(a)-m(b))\right)\right) \\
& +f_{i, a, b}\left(\frac{\|a\|-2^{i-1}}{2^{i-1}}\left(s_{i+1}(a)-s_{i+1}(b)-(m(a)-m(b))\right)\right) \\
& +(\|b\|-\|a\|) \cdot \frac{f_{i, a, b}\left(s_{i}(b)\right)}{2^{i-1}}+(\|a\|-\|b\|) \cdot \frac{f_{i, a, b}\left(s_{i+1}(b)\right)}{2^{i-1}} \\
\stackrel{44,(55, \& \llbracket 12]}{>} \frac{99}{1000}\|a-b\|-\frac{1}{1000}\|a-b\|-\frac{1}{100}\|a-b\| & \quad-4\|b\|-\|a\||-4|\|a\|-\|b\| \mid \\
= & \frac{88}{1000}\|a-b\|-8(\|a\|-\|b\|) .
\end{aligned}
$$

Now, as above, we consider the case when $\|a\|-\|b\|<\frac{1}{1000}\|a-b\|$ separately and complete the argument in the same way as above.

We turn to the subcase (13). Recall (see (3)) that $\left\|s_{i}(a)-s_{i}(b)\right\| \geq\|a-b\|$. Combining this with (13) and with the inequality $\|m(a)-m(b)\|<\frac{1}{100}\|a-b\|$, we get $\left\|s_{i}(a)-s_{i}(b)-(m(a)-m(b))\right\| \geq \frac{99}{100}\|a-b\|$ and $\frac{2^{i}-\|a\|}{2^{i-1}}<\frac{10}{99}$. (In the same way we get the inequality $\left\|s_{i+1}(a)-s_{i+1}(b)-(m(a)-m(b))\right\| \geq \frac{99}{100}\|a-b\|$, which we use below.) Therefore $\frac{\|a\|-2^{i-1}}{2^{i-1}}>\frac{89}{99}$. Applying the triangle inequality, we get

$$
\begin{aligned}
\|\varphi(a)-\varphi(b)\| \geq & \left\|\frac{\|a\|-2^{i-1}}{2^{i-1}}\left(s_{i+1}(a)-s_{i+1}(b)-(m(a)-m(b))\right)\right\| \\
& -\left\|\frac{2^{i}-\|a\|}{2^{i-1}}\left(s_{i}(a)-s_{i}(b)-(m(a)-m(b))\right)\right\| \\
& -\|m(a)-m(b)\|-8(\|a\|-\|b\|) \\
> & \frac{89}{99} \cdot \frac{99}{100}\|a-b\|-\frac{1}{10}\|a-b\|-\frac{1}{100}\|a-b\|-8(\|a\|-\|b\|) \\
= & \frac{78}{100}\|a-b\|-8(\|a\|-\|b\|) .
\end{aligned}
$$

Now, as was done twice already, we consider the case when $\|a\|-\|b\|<\frac{1}{100}\|a-b\|$ separately and complete the argument in the same way as above. This completes the argument in Case 1.

2.2. Case 2: $2^{i-1} \leq\|b\| \leq 2^{i} \leq\|a\| \leq 2^{i+1}$. We have

$$
\begin{aligned}
\varphi(a)-\varphi(b)= & -\frac{2^{i}-\|b\|}{2^{i-1}} s_{i}(b) \\
& +\frac{2^{i+1}-\|a\|}{2^{i}} s_{i+1}(a)-\frac{\|b\|-2^{i-1}}{2^{i-1}} s_{i+1}(b) \\
& +\frac{\|a\|-2^{i}}{2^{i}} s_{i+2}(a) .
\end{aligned}
$$


Estimate from above. The first and the last terms have norms $\leq 4(\|a\|-\|b\|)$. The norm of the two remaining terms can be estimated as follows:

$$
\begin{aligned}
& \left\|\frac{2^{i+1}-\|a\|}{2^{i}} s_{i+1}(a)-\frac{\|b\|-2^{i-1}}{2^{i-1}} s_{i+1}(b)\right\| \\
& \quad=\left\|\frac{2^{i}-\left(\|a\|-2^{i}\right)}{2^{i}} s_{i+1}(a)+\frac{\left(2^{i}-\|b\|\right)-2^{i-1}}{2^{i-1}} s_{i+1}(b)\right\| \\
& \quad=\left\|\left(s_{i+1}(a)-s_{i+1}(b)\right)-\frac{\left(\|a\|-2^{i}\right)}{2^{i}} s_{i+1}(a)+\frac{\left(2^{i}-\|b\|\right)}{2^{i-1}} s_{i+1}(b)\right\| \\
& \quad \leq 2\|a-b\|+4\left(\|a\|-2^{i}\right)+4\left(2^{i}-\|b\|\right) \leq 6\|a-b\| .
\end{aligned}
$$

Estimates from below. Rewriting and estimating some of the terms as in (16) we get

$$
\begin{aligned}
& \|\varphi(a)-\varphi(b)\| \\
& \geq\left\|\left(s_{i+1}(a)-s_{i+1}(b)\right)-\frac{\left(\|a\|-2^{i}\right)}{2^{i}} s_{i+1}(a)+\frac{\left(2^{i}-\|b\|\right)}{2^{i-1}} s_{i+1}(b)\right\| \\
& -\frac{2^{i}-\|b\|}{2^{i-1}}\left\|s_{i}(b)\right\|-\frac{\|a\|-2^{i}}{2^{i}}\left\|s_{i+2}(a)\right\| \\
& \geq\left\|s_{i+1}(a)-s_{i+1}(b)\right\|-12(\|a\|-\|b\|) \geq\|a-b\|-12(\|a\|-\|b\|),
\end{aligned}
$$

where in the last line we used (3). We complete the proof in this case as three times before. If $\|a\|-\|b\|<\frac{1}{20}\|a-b\|$, we get an estimate from (17). Otherwise we use (11).

2.3. Case 3: $2^{i-1} \leq\|b\| \leq 2^{i}<2^{k-1} \leq\|a\| \leq 2^{k}$. In this case we have

$$
3\left(2^{k}+2^{i}\right) \geq 3(\|a\|+\|b\|) \geq\|\widetilde{\varphi}(a)-\widetilde{\varphi}(b)\| \geq\|a\|-\|b\| \geq 2^{k-1}-2^{i} .
$$

Since

$$
\frac{3\left(2^{k}+2^{i}\right)}{2^{k-1}-2^{i}} \leq \frac{3 \cdot 2^{k+1}}{2^{k-2}} \leq 24
$$

and

$$
\|a\|+\|b\| \geq\|a-b\| \geq\|a\|-\|b\|,
$$

it follows that $\widetilde{\varphi}$ is bilipschitz.

2.4. Completion of the proof for spaces nonisomorphic to their hyperplanes. We have proved Theorem 1.2 in the case when $X$ is isomorphic to its hyperplane. To prove Theorem 1.2 in the general case we find a Lipschitz map $\tau: \mathbb{R}_{+} \rightarrow X$ such that the map $\hat{\varphi}: N \rightarrow X$ given by $\hat{\varphi}(a)=\tau(\|a\|)+\varphi(a)$ works just in the same way as $\widetilde{\varphi}$. It is easy to see that for this to be true we need the inequality

$$
\|\widehat{\varphi}(a)-\widehat{\varphi}(b)\| \geq \alpha(\|a\|-\|b\|)
$$

to hold for some $\alpha>0$. We rewrite this inequality as

$$
\|\tau(\|a\|)-\tau(\|b\|)+(\varphi(a)-\varphi(b))\| \geq \alpha(\|a\|-\|b\|) .
$$

Let $T_{i}=\left\{\varphi(u): u \in N,\|u\| \leq 3^{i+1}\right\}$. It is clear that all these sets are finite. We construct inductively a sequence $\left\{F_{i}\right\}_{i=1}^{\infty}$ of finite-dimensional subspaces of $X$ and a sequence $\left\{p_{i}\right\}_{i=1}^{\infty}$ of vectors. We let $F_{1}=\operatorname{lin}\left(T_{1}\right)$. Since $X$ is infinitedimensional (see the assumption made after Observation [2.2), there is $p_{1} \in S_{X}$ 
such that $\operatorname{dist}\left(p_{1}, F_{1}\right)=1$. Let $F_{2}=\operatorname{lin}\left(T_{2} \cup\left\{p_{1}\right\}\right)$ and $p_{2} \in S_{X}$ be such that $\operatorname{dist}\left(p_{2}, F_{2}\right)=1$. Letting $F_{3}=\operatorname{lin}\left(T_{3} \cup\left\{p_{i}\right\}_{i=1}^{2}\right)$, we continue in an obvious way.

We introduce the map $\tau: \mathbb{R}_{+} \rightarrow X$ in the following way:

$$
\tau(t)= \begin{cases}t p_{1} & \text { if } 0 \leq t \leq 3^{1} \\ 3^{1} p_{1}+\left(t-3^{1}\right) p_{2} & \text { if } 3^{1} \leq t \leq 3^{2} \\ 3^{1} p_{1}+\left(3^{2}-3^{1}\right) p_{2}+\left(t-3^{2}\right) p_{3} & \text { if } 3^{2} \leq t \leq 3^{3} \\ \cdots & \cdots \\ 3^{1} p_{1}+\left(3^{2}-3^{1}\right) p_{2} \cdots+\left(3^{k}-3^{k-1}\right) p_{k}+\left(t-3^{k}\right) p_{k+1} & \text { if } 3^{k} \leq t \leq 3^{k+1} \\ \cdots & \cdots\end{cases}
$$

Since $\left\|p_{i}\right\|=1$, the map $\tau$ is 1 -Lipschitz. It remains to show that the inequality (18) holds. We consider three cases:

(1) $3^{i} \leq\|b\| \leq\|a\| \leq 3^{i+1}$. The argument used in this case can also be used in the case $0 \leq\|b\| \leq\|a\| \leq 3^{1}$. Minor adjustments of the other cases are needed if $0 \leq\|b\| \leq 3^{1} \leq\|a\|$.

(2) $3^{i-1} \leq\|b\| \leq 3^{i} \leq\|a\| \leq 3^{i+1}$.

(3) $3^{k-1} \leq\|b\| \leq 3^{k} \leq 3^{i} \leq\|a\| \leq 3^{i+1}$, where $k<i$.

In the first case we have

$\|\tau(\|a\|)-\tau(\| b||)+(\varphi(a)-\varphi(b))\|=\left\|(\|a\|-\| b||) p_{i+1}+(\varphi(a)-\varphi(b))\right\| \geq\|a\|-\|b\|$.

The last inequality follows from $\operatorname{dist}\left(p_{i+1}, F_{i+1}\right)=\left\|p_{i+1}\right\|$ and $\varphi(a), \varphi(b) \in T_{i}$; therefore $\varphi(a)-\varphi(b) \in F_{i} \subset F_{i+1}$.

In the second case we consider two subcases:

$$
\begin{aligned}
& \|a\|-3^{i} \geq \frac{1}{3}(\|a\|-\|b\|), \\
& \|a\|-3^{i}<\frac{1}{3}(\|a\|-\|b\|) .
\end{aligned}
$$

In subcase (19) we get

$$
\begin{aligned}
\| \tau(\|a\|)-\tau(\|b\|) & +(\varphi(a)-\varphi(b)) \| \\
& =\left\|\left(\|a\|-3^{i}\right) p_{i+1}+\left(3^{i}-\|b\|\right) p_{i}+(\varphi(a)-\varphi(b))\right\| \\
& \geq\|a\|-3^{i} \geq \frac{1}{3}(\|a\|-\|b\|),
\end{aligned}
$$

where we use (19), $\operatorname{dist}\left(p_{i+1}, F_{i+1}\right)=\left\|p_{i+1}\right\|$, and $p_{i}, \varphi(a), \varphi(b) \in F_{i+1}$.

In subcase (20) we have

$$
\begin{aligned}
\| \tau(\|a\|)-\tau(\|b\|) & +(\varphi(a)-\varphi(b)) \| \\
& =\left\|\left(\|a\|-3^{i}\right) p_{i+1}+\left(3^{i}-\|b\|\right) p_{i}+(\varphi(a)-\varphi(b))\right\| \\
& \geq\left\|\left(3^{i}-\|b\|\right) p_{i}+(\varphi(a)-\varphi(b))\right\|-\left(\|a\|-3^{i}\right) \\
& \geq\left(3^{i}-\|b\|\right)-\left(\|a\|-3^{i}\right) \geq \frac{1}{3}(\|a\|-\|b\|) .
\end{aligned}
$$

In this chain of inequalities we use the fact that $\varphi(a), \varphi(b) \in T_{i}$, $\operatorname{dist}\left(p_{i}, F_{i}\right)=\left\|p_{i}\right\|$; in the last line we use the inequality $\|b\| \leq 3^{i} \leq\|a\|$ and (20).

Now we consider the third case. In this case we again consider subcases (19) and (20). In the first subcase the argument is exactly as above. So we focus on the 
second subcase. In this subcase we have

$$
\|a\|-3^{i}<\frac{1}{3}\left(3^{i}-3^{i-1}\right)
$$

see the defining inequality for the third case.

In this subcase we have

$$
\tau(\|a\|)-\tau(\| b||)=\left(\|a\|-3^{i}\right) p_{i+1}+\left(3^{i}-3^{i-1}\right) p_{i}+r,
$$

where $r$ is a vector contained in $F_{i}$. Thus

$$
\begin{aligned}
\| \tau(\|a\|)-\tau(\|b\|) & +(\varphi(a)-\varphi(b)) \| \\
& \geq\left\|\left(3^{i}-3^{i-1}\right) p_{i}+r+(\varphi(a)-\varphi(b))\right\|-\left\|\left(\|a\|-3^{i}\right) p_{i+1}\right\| \\
& \geq\left(3^{i}-3^{i-1}\right)-\frac{1}{3}\left(3^{i}-3^{i-1}\right)=\frac{4}{3} \cdot 3^{i-1} \geq \frac{4}{27}(\|a\|-\|b\|),
\end{aligned}
$$

where we use the fact that $r$ and $\varphi(a)-\varphi(b)$ are in $F_{i}$ and $\operatorname{dist}\left(p_{i}, F_{i}\right)=\left\|p_{i}\right\|$. This completes the proof of (18) and thus Theorem 1.2

\section{Proof in the COARSE CASE}

The proof of Theorem 1.2 contains almost everything we need for the proof of Theorem 1.3. We just need to modify the beginning of the proof.

Proof of Theorem 1.3. We pick a point $O$ in $A$ and let $A_{i}=\left\{a \in A: d_{A}(O, a) \leq\right.$ $\left.2^{i}\right\}$. By the assumption there are uniformly coarse maps $f_{i}: A_{i} \rightarrow X$. We may and shall assume that $f_{i}(O)=0$. Let $\mathcal{U}$ be a nontrivial ultrafilter on $\mathbb{N}$. The maps $\left\{f_{i}\right\}_{i=1}^{\infty}$ induce a map $f: A \rightarrow X^{\mathcal{U}}$ defined by $f(u)=\left\{\widetilde{f}_{i}(u)\right\}_{i=1}^{\infty}$, where

$$
\tilde{f}_{i}(u)= \begin{cases}f_{i}(u) & \text { if } u \in A_{i}, \\ 0 & \text { if } u \notin A_{i} .\end{cases}
$$

The definition of an ultraproduct immediately implies that $f: A \rightarrow X^{\mathcal{U}}$ is a coarse embedding. Letting $N=f(A)$, it is easy to check that $N$ with the metric induced from $X^{\mathcal{U}}$ is a locally finite metric space. The argument of the proof of Theorem 1.2 shows that there is a bilipschitz embedding of $N$ into $X$ (see Remark 2.1). Since the composition of a coarse and a bilipschitz embedding is a coarse embedding, the proof is completed.

\section{Relations With previous Results}

The main result of the paper [BL08] is

Theorem 4.1. If $X$ is a Banach space without cotype, then every locally finite metric space admits a bilipschitz embedding into $X$.

Theorem 4.1 is an immediate consequence of Theorem 1.2 and the following results (used in [BL08]):

- [Fre10, p. 161] (see also [Mat02, p. 385]): Each $n$-element metric space is isometric to a subset of $\ell_{\infty}^{n}$.

- [MP76]: The spaces $\left\{\ell_{\infty}^{n}\right\}_{n=1}^{\infty}$ admit uniformly bilipschitz embeddings into any Banach space $X$ without cotype.

The fact that Theorem 4.1 implies the following result of [BG05] was observed already in BL08]. 
Theorem 4.2 (BG05]). Let $A$ be a metric space with bounded geometry. There exists a sequence of positive real numbers $\left\{p_{n}\right\}$ and a coarse embedding of $A$ into the $\ell_{2}$-direct sum of $\left\{\ell_{p_{n}}\right\}_{n=1}^{\infty}$.

In Ost09 the following result was proved.

Theorem 4.3. Let $A$ be a locally finite metric space which admits a bilipschitz embedding into a Hilbert space, and let $X$ be an infinite-dimensional Banach space. Then there exists a bilipschitz embedding $f: A \rightarrow X$.

In Ost06b a coarse version of this result was proved. These results follow immediately from Theorems 1.2 and 1.3 and the Dvoretzky theorem Dvo61.

Theorem 1.2 can also be used to derive both of the main results of Bau07 from the finite versions of the results mentioned in Bau07.

\section{REFERENCES}

[Bau07] F. Baudier, Metrical characterization of super-reflexivity and linear type of Banach spaces, Archiv Math., 89 (2007), no. 5, 419-429. MR2363693(2009b:46019)

[BL08] F. Baudier, G. Lancien, Embeddings of locally finite metric spaces into Banach spaces, Proc. Amer. Math. Soc., 136 (2008), 1029-1033. MR2361878(2009c:46017)

[BG05] N. Brown, E. Guentner, Uniform embeddings of bounded geometry spaces into reflexive Banach space, Proc. Amer. Math. Soc., 133 (2005), no. 7, 2045-2050. MR.2137870 (2006b:57044)

[DK72] D. Dacuhna-Castelle, J.-L. Krivine, Applications des ultraproduits à l'étude des espaces et des algèbres de Banach, Studia Math., 41 (1972), 315-334. MR0305035 (46:4165)

[DJT95] J. Diestel, H. Jarchow, A. Tonge, Absolutely summing operators, Cambridge Studies in Advanced Mathematics, 43, Cambridge University Press, Cambridge, 1995. MR 1342297 (96i:46001)

[Dvo61] A. Dvoretzky, Some results on convex bodies and Banach spaces, in: Proc. Internat. Sympos. Linear Spaces (Jerusalem, 1960), pp. 123-160, Jerusalem Academic Press, Jerusalem; Pergamon, Oxford, 1961. MR0139079 (25:2518)

[Fre10] M. Fréchet, Les dimensions d'un ensemble abstrait, Math. Ann., 68 (1910), no. 2, 145168. MR 1511557

[GM93] W. T. Gowers, B. Maurey, The unconditional basic sequence problem, J. Amer. Math. Soc., 6 (1993), no. 4, 851-874. MR1201238(94k:46021)

[KP65] M. I. Kadets, A. Pełczyński, Basic sequences, bi-orthogonal systems and norming sets in Banach and Fréchet spaces (Russian), Studia Math., 25 (1965), 297-323. MR.0181886 $(31: 6112)$

[Mat02] J. Matoušek, Lectures on Discrete Geometry, Springer-Verlag, New York, 2002. MR 1899299 (2003f:52011)

[MP76] B. Maurey, G. Pisier, Séries de variables aléatoires vectorielles indépendantes et propriétés géométriques des espaces de Banach, Studia Math., 58 (1976), no. 1, 45-90. MR 0443015 (56:1388)

[Ost06a] M. I. Ostrovskii, Coarse embeddings of locally finite metric spaces into Banach spaces without cotype, C. R. Acad. Bulgare Sci., 59 (2006), no. 11, 1113-1116. MR 2293922 (2007m:46027)

[Ost06b] M.I. Ostrovskii, On comparison of the coarse embeddability into a Hilbert space and into other Banach spaces, unpublished manuscript, 2006, available at http:// facpub.stjohns.edu/ostrovsm

[Ost09] M. I. Ostrovskii, Coarse embeddability into Banach spaces, Topology Proc., 33 (2009), 163-183. MR 2471569(2010e:54021)

Department of Mathematics and Computer Science, St. John's University, 8000 UtoPIA Parkway, Queens, New York 11439

E-mail address: ostrovsm@stjohns.edu 